\title{
Optical TAMR Head Design for Placing a Heating Spot Close to a Magnetic Pole
}

\author{
K. Osawa ${ }^{* * *}$, K. Sekine ${ }^{* * *}$, M. Saka ${ }^{* * *}$, N. Nishida***, and H. Hatano* \\ *Business Development Department, Technology Development Headquarters, Konica Minolta Opto, Inc. \\ 2970 Ishikawa-machi, Hachioji Tokyo 192-8505, Japan \\ ${ }^{* *}$ Optics and Mechatronics R\&D Division, Device Technology R\&D Laboratories, Konica Minolta Technology Center, Inc. \\ ${ }^{* * *}$ MEMS Device R\&D Division, Device Technology R\&D Laboratories, Konica Minolta Technology Center, Inc.
} 1-2 Sakura-machi, Takatsuki-shi, Osaka 569-8503, Japan

We propose a new configuration for an optical thermally assisted magnetic recording (TAMR) head. The configuration allows us to place an optical near-field spot close to a magnetic write pole. In this system, we use a Focusing Waveguide integrated into a flying slider and a metal plasmon antenna attached to the bottom surface of the waveguide. With the proposed configuration, the intensity of the optical near-field spot peaks when the optical near-field spot is placed at the clad of the Focusing Waveguide. As a result, we can decrease the distance between the optical heating spot and the magnetic write pole. We present some simulation results of optical near-field generation. The distance between the near-field spot and the magnetic pole is decreased to $120 \mathrm{~nm}$. This configuration is useful in decreasing the time lag between optical heating and magnetic writing.

Key words: thermally assisted magnetic recording, spot size converter, optical waveguide, plasmon antenna

\section{Introduction}

In the field of magnetic recording, there has been considerable interest in thermally assisted magnetic recording (TAMR) systems for scaling the recording density beyond 1.55 Pbit/m² (1 Tbit/inch $\left.{ }^{2}\right){ }^{1)-8)}$. A heating spot with a diameter of less than $25 \mathrm{~nm}$ is required to achieve an areal density of over $1.55 \mathrm{Pbit} / \mathrm{m}^{2}$ for the TAMR system. One method of realizing a small heating spot on the recording medium is to use a near-field optical device. There have been many studies of integrated TAMR systems using near-field optical devices ${ }^{1)-6)}$. Previously, we proposed an optical TAMR head using a "Focusing Waveguide" (a waveguide-type spot size converter ${ }^{7)}$. In that paper, the design of the Focusing Waveguide was described and a focusing ratio of 10:1 was demonstrated. We also demonstrated the possibility of realizing optical near-field generation by using a plasmon antenna mounted on the bottom surface of the Focusing Waveguide.

One important issue for the TAMR system is to place the heating spot close to the magnetic pole, because a large offset between the heating spot and the magnetic pole may cause a degradation of the writability. To place the heating spot close to the magnetic pole, one can place the waveguide core close to the magnetic pole because, in general, the optical near-field is generated around the center of the waveguide core. However, the distance between the core and the magnetic pole is limited by the propagation loss due to the absorption of the magnetic pole ${ }^{1)}$. As will be describe in a later section, we estimated the limit to be about $300 \mathrm{~nm}$. This value is not sufficiently short for a
TAMR system.

In this paper, we propose a new configuration of a plasmon antenna. The design allows us to position the generation point of the optical near-field outside of the waveguide core, thus enabling the optical near-field to be placed very close to the magnetic pole. We have considered that the electric field distribution of the Focusing Waveguide might cause this particular phenomenon. We have demonstrated the new design by means of finite-difference time domain (FDTD) simulation. The TAMR head with the Focusing Waveguide is described in section 2. The simulation results are presented and discussed in section 3 ..

\section{Overview of the TAMR head design}

Figure 1 shows a schematic of the TAMR head with the Focusing Waveguide ${ }^{7)}$. Light irradiated from an optical fiber is focused by a lens element arranged between the fiber and a prism surface. The light is bent

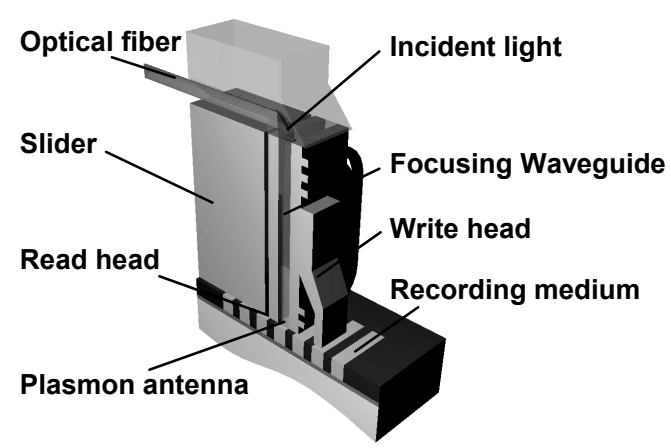

Fig. 1 Schematic of the TAMR head with the Focusing Waveguide. 


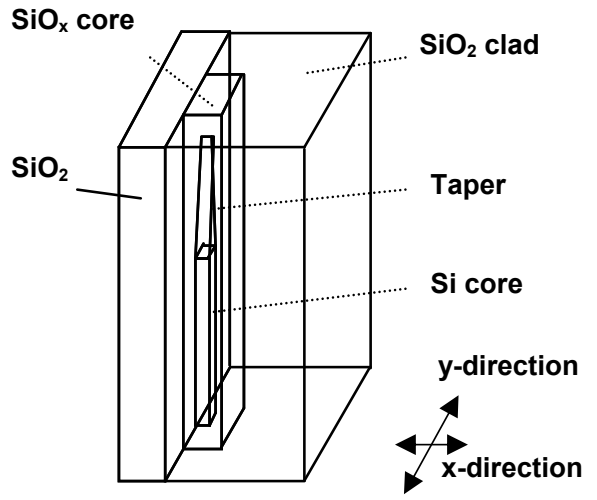

Fig. 2 Schematic of the the Focusing Waveguide.

at the prism surface and enters into the Focusing Waveguide which is integrated into a flying slider. The optical spot diameter of the light is reduced to about one tenth in the Focusing Waveguide. The sufficiently small optical spot then illuminates a plasmon antenna mounted on the bottom surface of the Focusing Waveguide. The plasmon antenna generates an optical near-field of $30 \mathrm{~nm}$ in diameter, which is used as the light source of the TAMR system.

Figure 2 shows the structure of the Focusing Waveguide, which consists of three parts, namely, the silicon core, the $\mathrm{SiO}_{\mathrm{x}}$ core, and the clad made of $\mathrm{SiO}_{2}$. The optical spot diameter of the light is reduced while the light propagates through the waveguide from the $\mathrm{SiO}_{\mathrm{x}}$ core to the $\mathrm{Si}$ core because of the mode conversion due to the tapered structure of the $\mathrm{Si}$ waveguide. The Focusing Waveguide can convert the spot size from 4 $\mu \mathrm{m}$ to $0.4 \mu \mathrm{m}$ with a loss of less than $1 \mathrm{~dB}^{9}$ ). The typical taper length is $200 \mu \mathrm{m}$ and the loss of the waveguide depends on the taper length ${ }^{9}$.

\section{Simulation results and discussion}

\subsection{Minimum distance between the waveguide core and} the magnetic pole

First, we discuss the minimum distance between the waveguide core and the magnetic pole. The geometry of the waveguide core and the magnetic pole is shown in Fig. 3. The figure shows the bottom portion of Fig. 2 (the magnetic pole is not shown in Fig. 2 ). Since Fig. 3 represents the region near the bottom surface of the Focusing Waveguide, the light propagates only in the silicon core. To place the optical near-field spot close to the magnetic pole, the core must be placed as close as possible to the magnetic pole. However, the propagation loss of the waveguide will increase when we place the magnetic pole close to the waveguide core, because of the absorption of the magnetic pole ${ }^{1)}$. In order to determine the minimum distance, we calculated the propagation loss of the waveguide by means of the finite

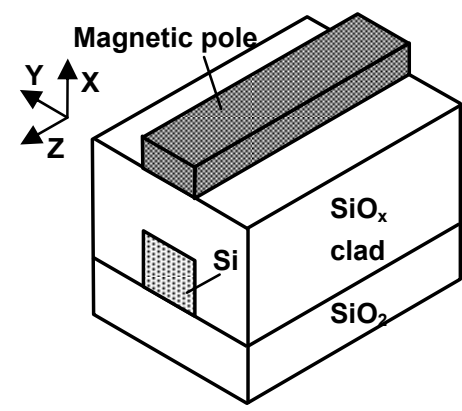

Fig. 3 Positions of the magnetic pole and waveguide.

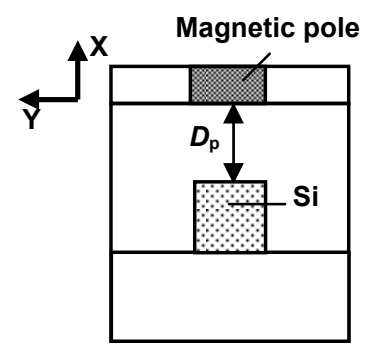

(a)

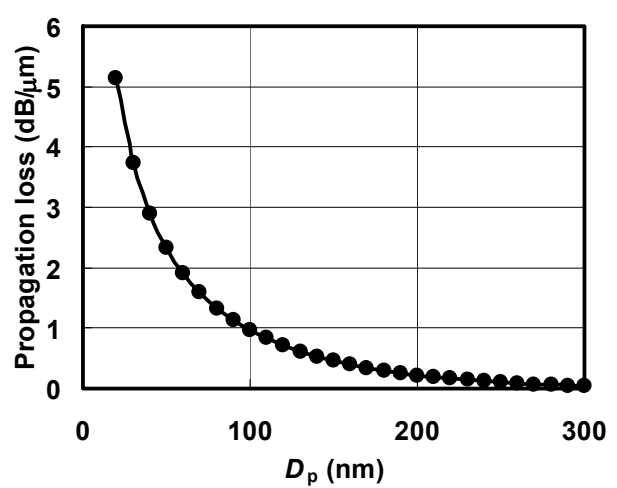

(b)

Fig. 4 Spacing dependence of the propagation loss of the waveguide. (a)Cross-section Propagation loss.

element method (FEM). Figure 4(a) shows a cross-section of the bottom surface of the Focusing Waveguide, which is used in the FEM simulation. In the figure, $D_{\mathrm{p}}$ represents the distance between the magnetic pole edge and the core edge. Figure 4(b) shows the calculated propagation loss as a function of $D_{\mathrm{p}}$. In this calculation, the material of the magnetic pole is assumed to be cobalt. The cross-sectional dimensions of the silicon core and the cobalt pole are assumed to be $300 \mathrm{~nm} \times 300 \mathrm{~nm}$ and $300 \mathrm{~nm} \times 150 \mathrm{~nm}$, respectively. The refractive indices of silicon and cobalt are taken to be 3.48 and $4.44+i 5.75$, respectively. The wavelength is assumed to be $1.5 \mathrm{\mu m}$. Figure 4(b) clearly shows a decrease in the propagation loss with an increase in $D_{\text {p }}$. The propagation loss decreases from $5.1 \mathrm{~dB} / \mu \mathrm{m}$ at $D_{\mathrm{p}}=$ $20 \mathrm{~nm}$ to $0.04 \mathrm{~dB} / \mu \mathrm{m}$ at $D_{\mathrm{p}}=300 \mathrm{~nm}$. To avoid a serious propagation loss, the minimum distance $D_{\text {pmin }}$ was assumed to be $300 \mathrm{~nm}$. 
3.2 New configuration for placing the heating spot close to the magnetic pole

To generate a small optical near-field spot, a plasmon antenna is mounted at the bottom surface of the Focusing Waveguide as shown in Fig. 5(a). In general, the optical near-field spot is generated efficiently around the center of the waveguide core. However, in that case, the minimum distance $D_{\text {pmin }}$ discussed in the previous section is not short enough for the TAMR system to use. Therefore, we designed a new configuration of a plasmon antenna by means of FDTD simulation. In this simulation, the distance between the core edge and the magnetic pole edge is set to $300 \mathrm{~nm}$ $\left(D_{\text {pmin }}\right)$. Since the effect of the magnetic pole is small at this distance, as shown in section 3.1, the magnetic pole

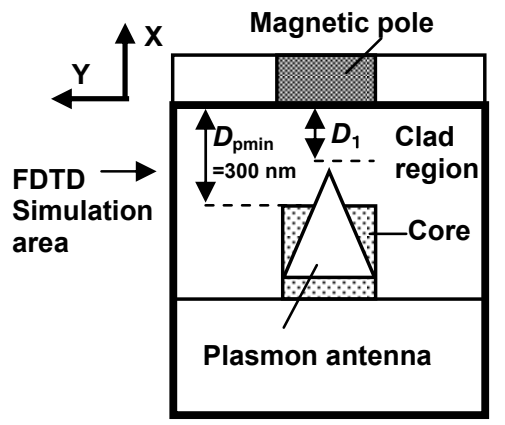

(a)

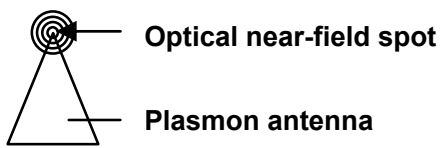

(b)

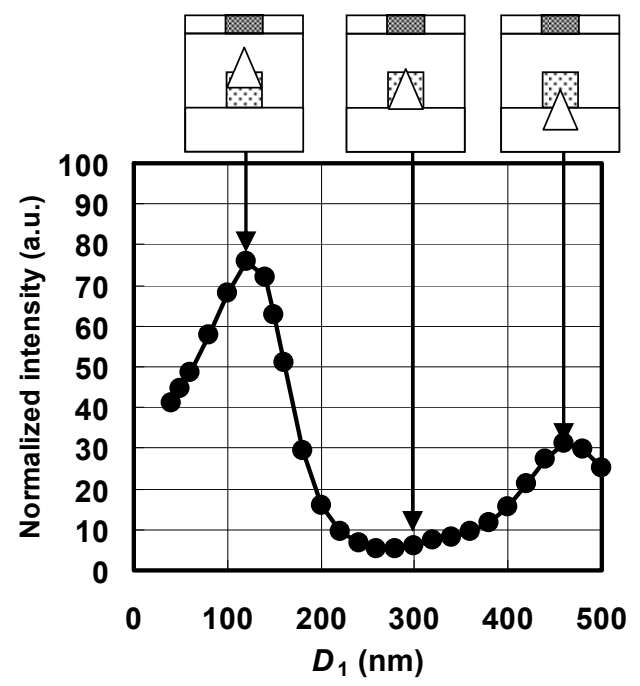

(c)

Fig. $5 \quad D_{1}$ dependence of the intensity of the optical near-field spot. (a) Position of the plasmon antenna. (b) Schematic of the optical near-field distribution. (c) Normalized intensity of the optical near-field spot as a function of $D_{1}$. is neglected in this FDTD calculation. The polarization direction is vertical to the substrate ( $\mathrm{x}$-polarization). The material of the plasmon antenna is gold, which has a broadband plasmon enhancement factor. The plasmon antenna is trianglular, and has a length of $300 \mathrm{~nm}$. The top angle and the thickness of the structure are $40^{\circ}$ and $40 \mathrm{~nm}$, respectively. The direction of the triangle is set to be the same as the polarization direction, in order to generate the optical near-field efficiently. The position of the plasmon antenna is defined by the distance between the apex of the triangle and the magnetic pole edge $\left(D_{1}\right) . D_{1}$ represents the distance between the heating spot and the magnetic writing spot, because the optical near-field spot is generated at the apex of the triangle, as shown in Fig.5(b). Figure 5(c) shows the intensity of the optical near-field spot as a function of $D_{1}$. The intensity of the optical near-field spot is normalized by the intensity, which is calculated without the plasmon antenna. In Fig. 5(c), the intensity of the optical near-field spot peaks at $D_{1}=120 \mathrm{~nm}$, where the optical near-field spot is generated in the clad region. Therefore, the heating spot is $330 \mathrm{~nm}$ from the center position of the waveguide core and approaches within $120 \mathrm{~nm}$ of the magnetic pole.

Figure 6 shows the distribution of the electric field intensity when $D_{1}$ is $120 \mathrm{~nm}$. Figure 6(a) represents the locations of the plasmon antenna and the optical near-field spot. Figures 6(b) and 6(c) show a cross-section of the electric field distribution at the peak position. The full-width at half maximum of the spot is $20 \mathrm{~nm}$ (in the $\mathrm{x}$-direction) and $30 \mathrm{~nm}$ (in the $\mathrm{y}$-direction). The spot size is small enough for a high-density TAMR system.

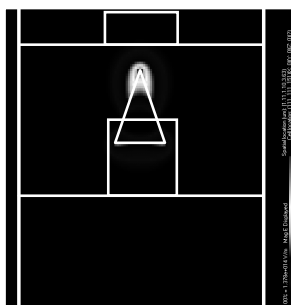

(a)

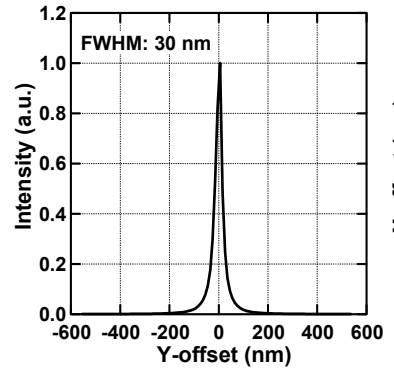

(b)

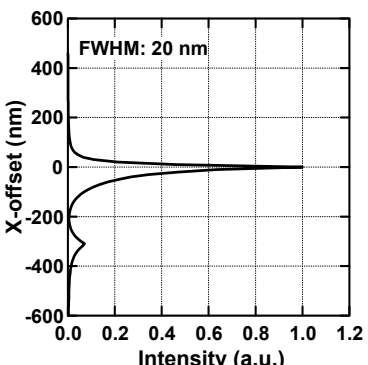

(c)
Fig. 6 Distribution of the electric field intensity when $D_{1}$ is $120 \mathrm{~nm}$. (a) Distribution of the electric field intensity. (b) Electric field intensity as a function of y-offset. (c) Electric field intensity as a function of $\mathrm{x}$-offset. 
As a result, it is confirmed that the heating spot can be placed very close to the magnetic pole by using our proposed configuration.

\subsection{Discussions}

Using the Focusing Waveguide, we can place the optical near-field spot close to the magnetic pole. This is most likely due to the electric field distribution of the Focusing Waveguide itself. To obtain a better interpretation, we calculated the electric field distribution of the bottom of the Focusing Waveguide by means of FEM. Figure 7 shows a cross-section of the bottom surface of the Focusing Waveguide. The wavelength is assumed to be $1.5 \mu \mathrm{m}$. The refractive indices of the $\mathrm{SiO}_{2}$ substrate, $\mathrm{Si}$ core, and $\mathrm{SiO}_{\mathrm{x}}$ clad are $1.44,3.48$, and 1.47 , respectively. The dimensions of the Si core are assumed to be $300 \mathrm{~nm} \times 300 \mathrm{~nm}$. The polarization direction is assumed to be vertical to the substrate ( $\mathrm{x}$-polarization). The main component of the electric field is in the $\mathrm{x}^{-}$direction $\left(E_{\mathrm{x}}\right)$. The simulation result is shown in Fig. 8. The electric field intensity peaks at the center of the core and at the boundary of the core and the clad. It is interesting to note that the peak of the electric field intensity occurs at the boundary of the core and the clad. This is due to the boundary condition of the electric flux density. Basically, as the difference in the refractive index between the core and the clad is increased, the electric field intensity near the boundary of the core and the clad should become larger. The Focusing Waveguide has a high refractive index difference between the Si core and the $\mathrm{SiO}_{\mathrm{x}}$ clad. Therefore, a strong electric field intensity occurs near the boundary of the core and the clad. If we place the plasmon antenna at the boundary of the core and the clad, we can apply a strong electric field to the plasmon antenna and generate a strong optical near-field spot. Using this configuration of the TAMR head, we can place the optical near-field spot close to the magnetic pole and generate a strong optical near-field spot.

\section{Conclusion}

We have proposed a new configuration of an optical TAMR head in order to place the optical heating spot close to the magnetic pole. Some simulations of the optical near-field spot generation have been described. This configuration is useful in decreasing the time lag between the optical heating and the magnetic writing. Using this configuration, we will be able to improve the writability of the TAMR system. For further work, it is necessary to confirm if the heat spot still be as small as the optical near-field spot size in the magnetic medium. A thermal simulation of the magnetic medium and an experimental demonstration are tasks for future work.

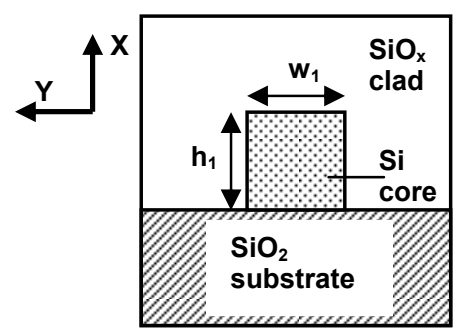

Fig. 7 Cross section of the Focusing Waveguide.

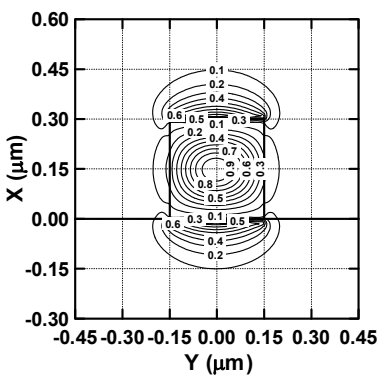

(a)

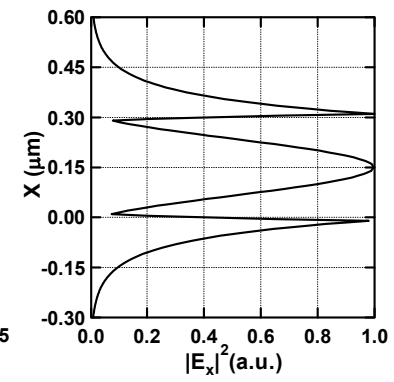

(b)
Fig. 8 Electric field distribution of the $x^{-c o m p o n e n t . ~(a) ~ M a p ~ o f ~ t h e ~ e l e c t r i c ~ f i e l d ~}$ intensity. (b) Cross-sectional view of $\mathrm{x}$-direction at $\mathrm{Y}=0$.

Acknowledgements A part of this paper is the property of the "Terabyte optical storage technology" project which OITDA contracted with The Ministry of Economy Trade and Industry of Japan (METI) in 2002 and contracted with The New Energy and Industrial Technology Development Organization (NEDO) since 2003 based on funds provided from METI.

\section{References}

1) M. A. Seigler, W. A. Challener, E. Gage, N. Gokemeijer, G. Ju, B. Lu, K. Pelhos, C. Peng, R. E. Rottmayer, X. Yang, H. Zhou, and T. Rausch: IEEE Trans. Magn., 44, 119 (2008).

2) W. A. Challener, C. Peng, A. V. Itagi, D. Karns, W. Peng, Y. Peng, X. Yang, X. Zhu, N. J. Gokemeijer, Y. T. Hsia, G. Ju, R. E. Rottmayer, A. Seigler, and E. C. Gage: Nature Photonics, 3, 303 (2009)

3) T. Matsumoto, Y. Anzai, T. Shintani, K. Nakamura, and T. Nishida: Opt. Lett., 31, 259 (2006).

4) M. Hirata, M. Oumi, K. Shibata, K. Nakajima, and T. Ohkubo, IEICE Trans. Electron., E90-C, 102 (2007).

5) S. Miyanishi, K. Innami, T. Naka, T. Kitazawa, M. Yagura, N. Teraguchi, Y. Murakami, and A. Takahashi, IEEE Trans. Magn., 44, 3360 (2008).

6) S. Miyanishi, N. Iketani, K. Takayama, K. Innami, I. Suzuki, T. Kitazawa, Y. Ogimoto, Y. Murakami, K. Kojima, and A. Takahashi: IEEE Trans. Magn., 41, 2817 (2005).

7) N. Nishida, H. Hatano, K. Sekine, K. Konno, M. Saka, and H. Ueda: J. Magn. Soc. Jpn., 32, 174 (2006).

8) F. Tawa, S. Hasegawa, and W. Odajima: J. Appl. Phys., 101, 09H503 (2007)

9) T. Shoji, T. Tsuchizawa, T. Watanabe, K. Yamada, and H. Morita: Electron. Lett., 38, 1670 (2002).

Received Jun. 01, 2009; Revised Aug. 08, 2009;

Accepted Aug. 14, 2009 